\title{
The TIR-NB-LRR Gene SNC1 Is Regulated at the Transcript Level by Multiple Factors
}

\author{
Yongqing Li, Shuhua Yang, Huijun Yang, and Jian Hua \\ Department of Plant Biology, Cornell University, Ithaca, NY 14850, U.S.A.
}

Submitted 21 March 2007. Accepted 6 July 2007.

\begin{abstract}
SNC1 (suppressor of NPR1, constitutive 1) is a haplotypespecific Toll and interleukin-1 receptor-like nucleotidebinding site leucine-rich repeat type of resistance $(R)$-like gene possibly mediating race-specific disease resistance. Inactivation of its negative regulator $\mathrm{BON1}(\mathrm{BONZAI1)/CPN1}$ and $B A P 1$ genes or upregulation of its expression epigenetically lead to constitutive defense responses and dwarf phenotype. Here, we report an autoactivation of $S N C 1$ by introducing it into Arabidopsis as a transgene. The $S N C 1$ genomic fragment confers a dwarf phenotype induced by defense response upregulation associated with a higher $S N C 1$ transcript level. Analysis of the $\beta$-glucuronidase reporter gene under the control of the $S N C 1$ promoter suggests three modes of regulation on the $S N C 1$ transcript level: a repression by the chromosomal structure, a feedback amplification from $S N C 1$ on its promoter sequences, and a repression by $B O N 1$. These regulations appear to be independent of each other. The regulation of $S N C 1$ possibly exemplifies a universally complex control of $\boldsymbol{R}$ genes to ensure a repression of $\boldsymbol{R}$ activation under nonstress conditions and a robust activation of defense responses once the $R$ gene is induced.
\end{abstract}

Race-specific pathogen resistance is mediated by an interaction between a plant disease resistance $(R)$ gene and its corresponding pathogen avirulence (Avr) gene (Flor 1971). The genefor-gene interaction often leads to defense responses, including hypersensitive response (HR), to restrict the growth of the pathogen (Hammond-Kosack and Jones 1996). A number of $R$ genes have been molecularly cloned in many species. In spite of the diverse array of pathogens they perceive, the $R$ genes mostly encode five classes of proteins, with $\mathrm{R}$ proteins in the largest family containing nucleotide-binding site (NB) and leucine-rich repeat (LRR) domains (Dangl and Jones 2001; Martin et al. 2003). The amino-termini of these proteins are of either the Toll and interleukin-1 receptor-like (TIR) type or the coiled-coiled (CC) type. There are 149 NB-LRR-encoding genes in the Columbia ( $\mathrm{Col}$ ) accession of Arabidopsis thaliana (Meyers et al. 2003). The biological functions of most of these genes have not been identified, but it generally is thought that they are used to detect a vast variety of pathogens. Recently, they also have been implicated in downstream signaling pathways in defense response (Peart et al. 2005).

Corresponding author: Jian Hua; E-mail: jh299@ cornell.edu

Current address of S. Yang: College of Biological Sciences, China Agricultural University, Beijing, China.

* The $\boldsymbol{e}$-Xtra logo stands for "electronic extra" and indicates that Figures $1,4,5,6$, and 7 appear in color online.
$R$ gene activities are highly regulated for optimal plant defense and plant growth. Recognition of cognate Avr proteins activates $\mathrm{R}$ proteins to induce downstream defense responses. The interaction between $\mathrm{R}$ and Avr could be direct, similar to a ligand-receptor interaction (Deslandes et al. 2003; Dodds et al. 2006; Jia et al. 2000). It also could be indirect, where an R protein is guarded by a plant guardee protein whose activity can be modulated by pathogen effector proteins (Belkhadir et al. 2004; Dangl and Jones 2001; Martin et al. 2003; Schneider 2002; van der Biezen and Jones 1998). The activity of R proteins also can be regulated at the intramolecular level. Structurefunction analysis indicates that the LRRs control the molecular state of the NB domain and NB might serve as a molecular switch which undergoes conformational changes upon pathogen recognition (Takken et al. 2006). Recent studies reveal another aspect of $\mathrm{R}$ protein regulation that involves subcellular compartmentation and protein-protein associations (Burch-Smith et al. 2007; Shen et al. 2007).

The level of $R$ gene transcripts is usually low under nonpathogen invasion conditions, presumably preventing growth inhibition caused by high expression of $R$ genes. Overexpression of $R$ genes has been shown to lead to induction of plant defense responses in the absence of pathogen invasion, and the resistance tends to be broad spectrum. This effect has been observed for Prf (Oldroyd and Staskawicz 1998), Pto (Tang et al. 1999), and Cf-9 (Wulff et al. 2004). Constitutive activation of $R$ gene activity and defense response often are accompanied by dwarfism and sometimes lethality, a fitness cost underlying the necessity for tight regulation of $R$ genes (Heil and Baldwin 2002). $R$ genes can be modulated at the transcript level. Salicylic acid can induce the expression of a number of $R$ genes including SSI4, RPW8, and SNC1 (suppressor of NPR1, constitutive 1), possibly acting as an amplification of defense response (Shirano et al. 2002; Xiao et al. 2003; Yang and Hua 2004). The transcript level of $R$ gene also has been found to be regulated by pathogen. A rice $R$ gene, $X a 27$, encoding a small protein without significant motifs is shown to be induced by its cognate AvrXa27 effector protein, and its inducibility appears to determine the resistance or susceptibility of the rice cultivar ( $\mathrm{Gu}$ et al. 2005).

SNC1 $(B A L)$ is a TIR-NB-LRR type of $R$ gene residing in the RPP5 gene cluster that is very divergent among accessions in Arabidopsis (Noel et al. 1999; Stokes et al., 2002; Zhang et al. 2003). The RPP4 and RPP5 genes are the first genes of this cluster from the $\mathrm{Col}$ and the Landsberg erecta (Ler) accessions, respectively, and they are responsible for resistance to different variants of the downy mildew (Parker et al. 1997; van der Biezen et al. 2002). SNC1 is the second gene in the Col cluster and no pathogen specificity has been assigned to this gene. However missense mutation in the sncl-1 mutant induces constitutive defense responses likely 
due to an activation of the SNC1 protein (Li et al. 2001; Zhang et al. 2003). An epigenetic allele of SNC1, namely bal or snc1-2, exhibits a higher expression of $S N C 1$ than the wild type and also leads to constitutive defense responses and a dwarf phenotype (Stokes et al. 2002). These observations suggest that $S N C 1$ is a functional $R$ gene that can induce defense responses when activated.

Intriguingly, $S N C 1$ is under a negative control of the Arabidopsis BON1/CPN1 gene and its functional partner BAP1 gene (Yang and Hua 2004; Yang et al. 2006). BON1 is an evolutionarily conserved copine gene and the loss-offunction bon 1 mutants have enhanced disease resistance and a dwarf phenotype in a temperature- and humidity-dependent manner (Hua et al. 2001; Jambunathan et al. 2001). BAPl encodes a small lipid-binding protein and its loss-of-function mutants have a phenotype similar to but weaker than the bonl mutants (Yang et al. 2006). In the bonl and bapl lossof-function mutants, the SNC1 gene presumably is activated, leading to constitutive defense responses and reduced plant growth (Yang and Hua 2004; Yang et al. 2006). SNC1 transcript is higher in bon 1-1 and bap1-1 than in the wild type (Yang and Hua 2004; Yang et al. 2006). However, it is not known whether the repression of $S N C 1$ by BON1 and BAP1 is primarily at the level of the $S N C 1$ transcript or the SNC1 protein activity. $S N C l$ is shown to be regulated at the transcript level by temperature and salicylic acid (SA). There is a lower expression of $S N C 1$ at 28 than at $22^{\circ} \mathrm{C}$ and SA application induces the level of its transcript (Yang and Hua 2004). The SNCl transcript level appears to be regulated epigenetically as well. The mutant phenotype of bal/sncl-2 isolated from the $d d m 1$ background was due to an overexpression of SNC1, but there was no nucleotide change identified in the SNC1 gene (Stokes et al. 2002). Ethyl methane sulfonate (EMS) mutagenesis of bal induced a high incidence of reversion, further indicating that $S N C 1$ is under an epigenetic control (Stokes et al. 2002).

In this study, we further investigated the regulation of the $R$ gene $S N C 1$. We found that $S N C l$ as a transgene has a much higher expression than the endogenous gene, and this confers a dwarf phenotype as a consequence of upregulation of defense responses. Using transgenic plants with the SNC1 promoterreporter gene fusion, we further demonstrated a repression of SNC1 transcript level at the endogenous location and a regulation of SNC1 by BONI and SNC1 through the promoter of SNC1.
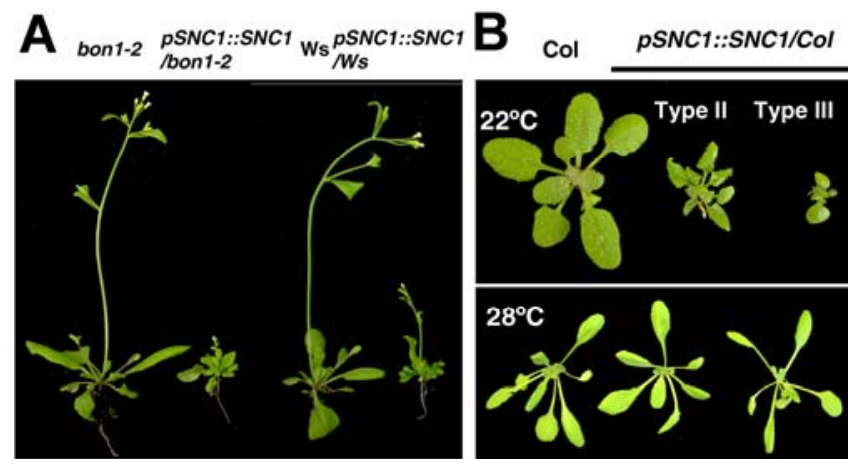

Fig. 1. $p S N C 1:: S N C 1$ confers a dwarf phenotype. A, Growth phenotypes of pSNC1::SNC1 transgenic plants in bon1-2 and the wild-type Ws at $22^{\circ} \mathrm{C}$. Shown are representative transgenic lines next to the nontransgenic line. B, Growth phenotypes of $p S N C 1:: S N C 1$ transgenic plants in the Col0 background. At $22^{\circ} \mathrm{C}$, hemizygous transgenic plants had a bon1-1-like phenotype and homozygous transgenics had a more severe phenotype. At $28^{\circ} \mathrm{C}$, they both showed wild-type phenotypes.

\section{RESULTS}

\section{Transgenic plants}

with pSNC1::SNC1 exhibit dwarf phenotype.

SNC1 is shown to mediate the dwarf phenotype of the bon-1 mutant in the Arabidopsis accession Col-0, and the bonl-2 mutant in the Ws accession does not exhibit an obvious growth defect due to a lack of functional SNC1 (Yang and Hua 2004). We hypothesize that introducing the wild-type $S N C 1$ gene to bon 1-2 should recapitulate the bon 1-1 dwarf phenotype. To test this, we isolated the genomic fragment of the SNCl gene starting from $1,912 \mathrm{bp} 5^{\prime}$ to the ATG translation start site and ending at $1,005 \mathrm{bp} 3^{\prime}$ to the stop codon and transformed this pSNC1::SNC1 fragment into the wild-type Ws and bon1-2. As anticipated, most of the T1 plants (16 of 25) of pSNC1::SNC1 in bon1-2 exhibited a bon1-1-like phenotype, including dwarfed stature and curled leaves with water-soaked appearance (Fig. 1A). Surprisingly, introduction of $p S N C 1:: S N C 1$ into the wild-type Ws plants caused a mutant phenotype as well. In all, 10 of 25 independent T1 plants exhibited a bon11-like phenotype (Fig. 1A). We subsequently introduced pSNC1::SNC1 into the wild-type Col background. Of the 17 T1 plants, 15 exhibited dwarf phenotype to various degrees (Fig. 1B). Thus, $p S N C 1:: S N C 1$ is capable of conferring a dwarf phenotype to wild-type plants independent of the repression function of $B O N 1$.

The progenies of $p S N C 1:: S N C 1$ primary transformants in Col gave rise to three types of phenotype: wild type, bon 1-1like, and extremely dwarf (Fig. 1B). Wild-type-looking plants were not transgenic, confirmed by sensitivity of their progenies to antibiotics used for transgene selection. The bon1-1like plants were similar in phenotype to their parents-small in stature, with curly and water-soaked leaves-but were fertile. They gave rise to progenies with all three types of phenotype and, thus, likely are hemizygous for the transgene. The extreme dwarf plants were arrested in growth at an early seedling stage and they died before bolting when grown at $22^{\circ} \mathrm{C}$ but grew like the wild type at $28^{\circ} \mathrm{C}$ (Fig. 1B). They gave rise to progenies with a uniform extreme dwarf phenotype, indicating that they are homozygous for the transgene. Thus, the severity of the phenotype is correlated with the zygosity or copy number of the transgene.

\section{pSNC1::SNC1 transgenic plants have enhanced disease resistance.}

We reasoned that the dwarf phenotype of pSNC1::SNC1 transgenics likely results from a constitutive defense response because it is very similar to that of bon 1-1, snc1-1, and snc1-2 (Stokes et al. 2002; Yang and Hua 2004). To test this hypothesis, we compared the growth of virulent Pseudomonas syringae pv. tomato DC3000 in the transgenics with that of the wild-type Col. At 3 days after dip inoculation, $P$. syringae pv. tomato DC3000 grew to $10^{8}$ while five independent pSNC1::SNC1 lines grew to $10^{4}$ to $10^{5}$ respectively (Fig. 2A). The transgenics were as resistant as bonl-1 that had $10^{5}$ growth of the pathogen (Fig. 2A). Consistent with the disease resistance phenotype, we found that the $p S N C 1:: S N C 1$ transgenics had a higher expression of the pathogenesis-related 1 $(P R 1)$ gene, a molecular marker for defense response (Uknes et al. 1992) (Fig. 2B).

To further test the hypothesis that the dwarf phenotype results from constitutive defense responses, we crossed a hemizygous $p S N C 1: S N C 1 / \mathrm{Col}$ transgenic into a Col plant containing a bacterial $N a h G$ gene encoding an SA-degrading salicylate hydroxylase (Lawton et al. 1995). F1 plants with $p S N C 1:: S N C 1$ (hemizygous) containing $N a h G$ exhibited a much weaker phenotype than hemizygous transgenics without nahG (data not 
shown). Consistently, the upregulation of $P R I$ expression in pSNC1::SNC1 transgenics was suppressed by $N a h G$ (Fig. 2B). Thus, the dwarf phenotype likely results from activated defense responses mediated by SA.

\section{pSNC1::SNC1 transgenic plants have}

a high expression of $S N C 1$.

We analyzed the transcript level of SNC1 in pSNC1::SNC1 transgenic lines by RNA blot hybridization and found that it was elevated in plants with dwarf phenotypes (Fig. 3A). The SNCl transcript level is low in the wild-type Col plants but is elevated in bon1-1. Hemizygous transgenic plants, with a bon1-1 like phenotype, had an SNC1 transcript level similar to that of bon1-1. Type III plants, with a more severe phenotype, had the highest SNC1 transcript level. Thus, the degree of the morphological defect is correlated with the level of the SNCl RNA transcript. More SNC1 transcript is accumulated in the homozygous plants with a more severe phenotype than in the hemizygous plants.
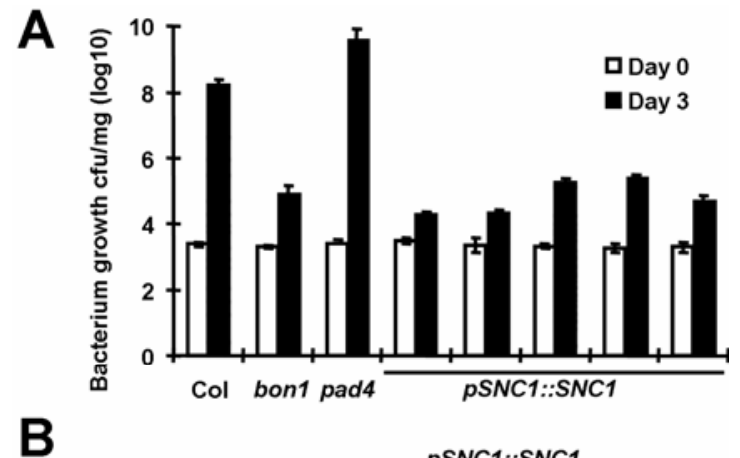

B

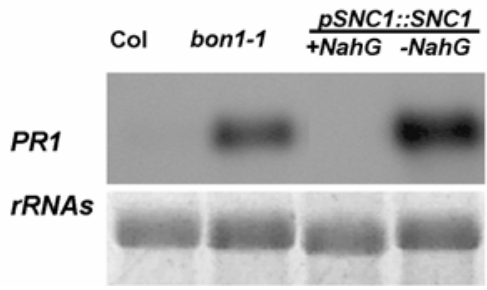

Fig. 2. $p S N C 1:: S N C 1$ transgenic plants have enhanced resistance to a virulent pathogen. A, Growth of Pseudomonas syringae pv. tomato DC3000 in Col-0, bon1-1, pad4-1, and five independent pSNC1::SNC1 transgenic lines. pad4 is more susceptible to the pathogen than Col, while all five lines and bonl are more resistant than Col. The data shown is from one of two biological repeats. B, pSNC1::SNC1 transgenic plants have upregulated $P R 1$ expression. RNA blot analysis shows that $P R 1$ is upregulated in the $p S N C 1:: S N C 1$ transgenic compared with the wild type, but this upregulation is abolished with NahG that degrades salicylic acid. $r R N A$ s are used as controls for equal loading (same in subsequent RNA blot analyses).
The fact that $p S N C 1:: S N C 1 / \mathrm{Col}$ has a very high $S N C 1$ transcript level prompted us to determine whether these lines contain high copies of transgenes, as was reported for the $R P W 8$ transgenics (Xiao et al. 2003). We carried out a Southern blot analysis on three $p S N C 1:: S N C 1 / \mathrm{Col}$ transgenic lines. A $S N C 1$-specific probe revealed two fragments, one representing the endogenous $S N C 1$ at $3.8 \mathrm{~kb}$ and the other representing the transgene at $2.2 \mathrm{~kb}$. Using the program ImageJ, we quantified SNC1 signals on the blot. The signal ratios of transgene versus the endogenous gene were $2.1,1.4$, and 1.9 for line no. 1, no. 2, and no. 11, respectively (Fig. 3B). Because these three lines likely only contain one or two pSNC1::SNC1 transgenes, the dwarf phenotype does not require very high copy numbers of the SNC1 gene. Furthermore, the mutant phenotype is unlikely due to having three copies of SNC1 (two endogenous copies and one transgene copy) in transgenics rather than two copies in Col because pSNC1::SNC1 conferred a dwarf phenotype in the Ws background. Some T1 transgenics in Ws or bon1-2 would have only one copy of $S N C l$ because there is no endogenous $S N C 1$, and yet they still exhibited dwarf phenotypes.

\section{pSNC1::SNC1:GFP transgenic plants reveal a feedback regulation \\ on both the transgene and the endogenous gene.}

We hypothesize that the high expression of the pSNC1::SNC1 transgene is partly due to a feedback regulation from activated defense responses. To test this, we constructed a chimeric gene $p S N C 1:: S N C 1: G F P$ similar to $p S N C 1:: S N C 1$, except that a green fluorescent protein (GFP) is fused in frame to the carboxyl terminus of SNC1. Transgenics of pSNC1:: SNC1:GFP were generated in the Col background and some of them exhibited a dwarf phenotype at $22^{\circ} \mathrm{C}$, similar to the pSNC1::SNC1 transgenics, and this dwarf phenotype could be suppressed by a higher growth temperature of $28^{\circ} \mathrm{C}$ (Fig. 4A). This suggests that $p S N C 1:: S N C 1: G F P$ has an activity similar to $p S N C 1:: S N C 1$ and it is possible to distinguish the endogenous $S N C 1$ from the $S N C 1$ transgene in $p S N C 1:: S N C 1: G F P$ transgenics.

We analyzed the expression of the endogenous $S N C 1$ and the pSNC1::SNC1:GFP transgene in transgenic plants. We found they both had a higher expression in transgenics than the endogenous gene in the wild type at $22^{\circ} \mathrm{C}$ (Fig. 4B). Thus, the presence of the transgene increases the expression of the endogenous SNCl gene. However, the endogenous SNC1 was expressed at a similar level in the transgenic plants and the wild type at $28^{\circ} \mathrm{C}$ (Fig. 4B). At this higher temperature, the transgenic plants were wild-type looking, presumably because of a suppression of defense responses. Thus, the upregulation of the endogenous and the transgene
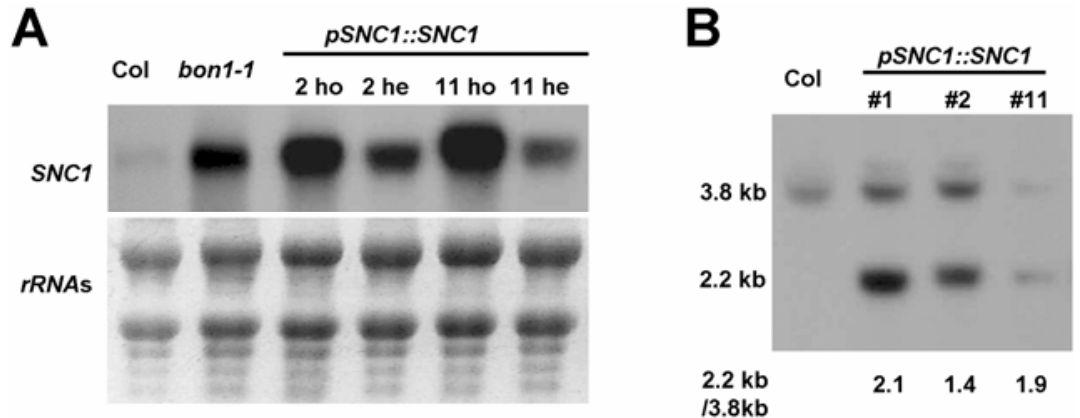

Fig. 3. $p S N C 1:: S N C 1$ transgenic plants have a high $S N C 1$ expression level. A, $S N C 1$ transcript levels in the wild-type Col, bon1-1, and transgenic lines no. 2 and no. 11 in homozygous (ho) and hemizygous (he) status. B, Southern blot analysis of the genomic DNAs of three $p S N C 1:: S N C 1$ transgenic lines. The 3.8$\mathrm{kb}$ fragment represents the endogenous $S N C 1$ gene and the $2.2-\mathrm{kb}$ fragment represents the transgene. Signal ratios of the two genes are indicated. 
SNC1 at $22^{\circ} \mathrm{C}$ likely is due to a feedback from the activation of defense responses. This is further supported by higher transcript levels of $S N C 1: G F P$ and $S N C 1$ at $22^{\circ} \mathrm{C}$ compared with $28^{\circ} \mathrm{C}$ (Fig. 4B).

\section{pSNC1::GUS transgene has a higher expression than the endogenous $S N C 1$.}

We noted that the SNC1:GFP transgene had a higher expression than the endogenous $S N C 1$ at $28^{\circ} \mathrm{C}$, suggesting that a repression of expression at the endogenous $S N C 1$ location is released in the transgene location. We hypothesize that an initial increase of the $S N C 1$ transcript level in transgenic plants is amplified through feedback regulation from defense response activation, leading to further increase of the $S N C 1$ transcript level.

To further test this hypothesis, we made a fusion construct driving the $\beta$-glucuronidase (GUS) reporter gene by the promoter and the first exon (encoding approximately $1 / 10$ of the protein) of $S N C 1$. This $p S N C 1:: G U S$ was introduced into Col and more than 18 transgenic lines were generated, and they all showed wild-type phenotypes. The GUS gene was found to be ubiquitously expressed in 15 of 18 lines, and young leaves and stems appeared to have slightly higher activities than older tissues (Fig. 5A). No GUS activity was observed in flowers except for the sepals.

Two representative lines with a single T-DNA insertion (determined by transgene segregation) were chosen for further analysis. We compared the transcript level of the SNCl endogenous gene and the $p S N C 1:: G U S$ transgene by RNA blot analysis with a probe complementary to the first exon of SNC1. Consistent with the hypothesis, the pSNC1::GUS transgene had a much higher expression level than the endogenous SNCl gene (data of one line is shown in Figure 5B). These data, in conjunction with a higher expression of pSNC1::SNC1:GFP compared with SNC1 at $28^{\circ} \mathrm{C}$ (Fig. 4B), suggest that there is a repression of the SNCl gene at the transcriptional level in its endogenous location.

A

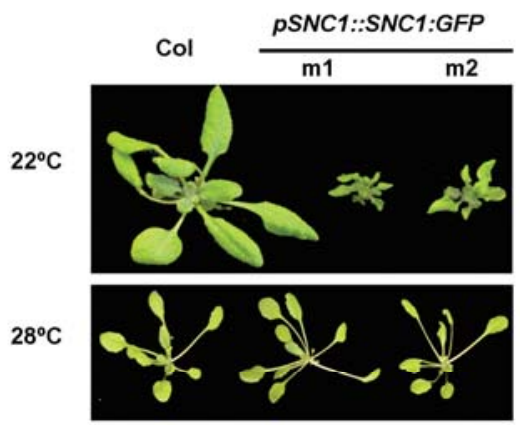

B

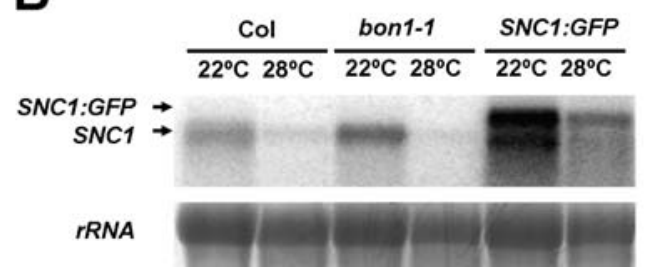

Fig. 4. $S N C 1$ is upregulated through a feedback regulation. A, $p S N C 1:$ : SNC1: GFP transgenic plants have a dwarf phenotype at $22^{\circ} \mathrm{C}$ but not $28^{\circ} \mathrm{C}$. On the left is a wild-type $\mathrm{Col}$ plant and on the right are the two representative transgenic lines ( $\mathrm{m} 1$ and $\mathrm{m} 2)$. B, $p S N C 1:: S N C 1: G F P$ transgenic plants have a higher $S N C 1$ and $S N C 1: G F P$ expression at 22 than at $28^{\circ} \mathrm{C}$. Notice that the endogenous $S N C 1$ expression at $22^{\circ} \mathrm{C}$ is higher in the transgenic than in the wild type.
Regulation of the pSNC1::GUS transgene by BON1.

$S N C 1$ is negatively regulated by $B O N 1$, possibly at the transcript level or protein activity level. To determine whether the $S N C 1$ transcription can be repressed by $B O N 1$, we compared the pSNC1::GUS expression in bonl-1 and the wild-type Col. More than 10 independent transgenic lines of $p S N C 1:: G U S$ were generated in the bon $1-1$ background and there appeared to be a higher expression of GUS in bonl-1 than in Col in general. However, the GUS activities exhibited variability among different lines likely due to positional effects of the transgene. To unambiguously compare the level of $p S N C 1:: G U S$ in the wild-type Col and bon 1-1, we generated near isogenic lines (NILs) with the same transgene in Col and bonl-1 backgrounds for three independent lines. This was achieved by crossing $p S N C 1:: G U S / C$ Col to bon $1-1$ and isolating transgenics in BON1 and bon1-1, respectively, through polymerase chain reaction (PCR) genotyping.

GUS staining indicates that there was a moderate upregulation of GUS activity in bon 1-1 compared with the wild-type Col (Fig. 6A). We confirmed this by RNA blot analysis on plants approximately 3 weeks old. The $p S N C 1:: G U S$ transgene had a higher transcript level in bon 1-1 than in Col, similar to the endogenous $S N C 1$ (Fig. 6B). Thus, the BON1 gene represses the transgene $p S N C 1: \because G U S$ in addition to the endogenous $S N C 1$ gene, and the promoter region of $S N C l$ appears to contain elements responsible for this repression.

We further asked whether the higher expression of $p S N C 1::$ GUS in bon1-1 is through a feedback from $S N C 1$, as suggested by a previous study on the endogenous SNC1 gene (Yang and Hua 2004). We introduced a loss-of-function snc1-11 mutation into transgenic lines and generated NILs of pSNC1::GUS in the wild-type Col, snc1-11, bon1-1, and bon1-1snc1-11. Southern blot analysis confirmed that all NILs of the four genotypes from the same T1 plant indeed contained the same transgene (data not shown). Presumably, feedback regulation from activation of defense responses is blocked by $s n c 1-11$, as indicated by the wild-type phenotype of bon1-1snc1-11. Consistent with GUS staining in these NILs (Fig. 6A), RNA blot analysis on 3-week-old plants revealed different levels of $p S N C 1: \because G U S$ transcript in different genetic backgrounds (Fig. 6B). Gene bon 1-1 had the highest expression of pSNC1::GUS; Col and bon1-1snc1-11 had comparable levels lower than that of bon1-1; and sncl-11 had the lowest expression. pSNC1::GUS has a higher expression

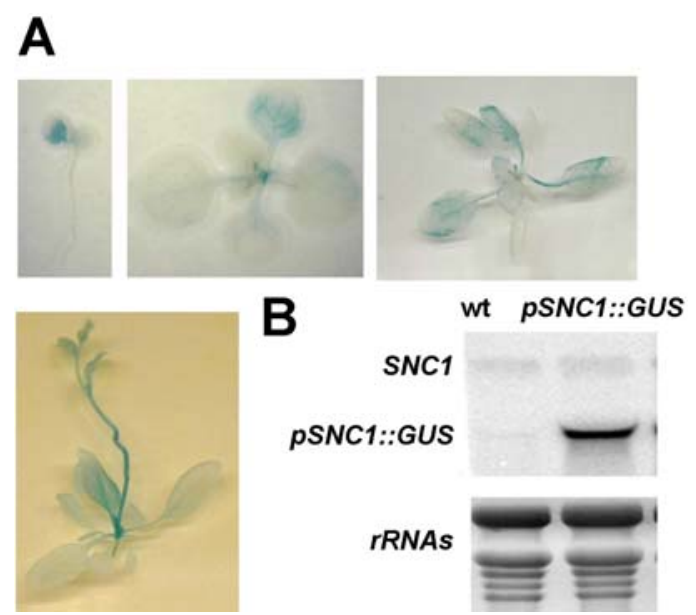

Fig. 5. pSNC1::GUS expression in transgenic plants. A, Expression of pSNC1::GUS at different developmental stages of a representative line assayed by $\beta$-glucuronidase (GUS) staining. B, pSNC1::GUS has a higher expression than the endogenous $S N C 1$ in a representative line. A probe is used to detect both $S N C 1$ and $S N C 1$ :GUS transcripts. 
in Col than in $s n c 1-11$, indicating that $S N C 1$ can feedback to regulate itself through its promoter. $p S N C 1:: G U S$ has a higher expression in bon $1-1$ even in the absence of a functional $S N C 1$, indicating that $B O N 1$ can regulate the promoter of SNC1 without a feedback regulation.

Interaction of different regulations on $\mathrm{SNC1}$ gene activity.

SNC1 activity is regulated by multiple modes at its endogenous locus: a transcriptional repression at the SNC1 locus (indicated by the $p S N C 1:: S N C 1$ and $p S N C 1:: G U S$ transgenic lines), epigenetic repression (as in bal and cprl), and protein activity (as in $s n c 1-1$ ). To determine whether BON1 regulates $S N C 1$ through one of these modes, we analyzed the interaction of the regulations by $B O N 1$ and by the $S N C 1$ locus.

We first compared the phenotypes of $p S N C 1:: S N C 1$ transgenic plants in bon1-2 and the wild-type Ws. For most of the lines, phenotypes were more severe in bonl-2 than in Ws, especially in inflorescence (Fig. 7A). Flowers of the pSNC1:: SNC1/bon1-2 transgenics were smaller than those of the pSNC1::SNC1/Ws transgenics, and they tended to open their sepals and petals at an earlier developmental stage than the wild type. These phenotypes frequently were observed in enhancers of bonl (unpublished data), indicating a stronger phenotype of $p S N C 1:: S N C 1 /$ bon 1-2.

Gene $5 n c 1-1$ has a missense mutation which might cause an activation of the SNC1 protein activity (Zhang et al. 2003). We generated a sncl-1bonl-1 double mutant, and found it had a more severe phenotype than either of the single mutants (Fig. 7B). At $22^{\circ} \mathrm{C}$, the double mutant was even smaller than the single mutants and grew like a tiny ball. At $28^{\circ} \mathrm{C}, s n c 1-1$ and bon $1-1$ grew like the wild type but the double mutant showed a dwarf phenotype.

Gene bal (sncl-2) is an epi-allele of SNC1 with a high expression of the SNC1 transcript (Stokes et al. 2002); whereas
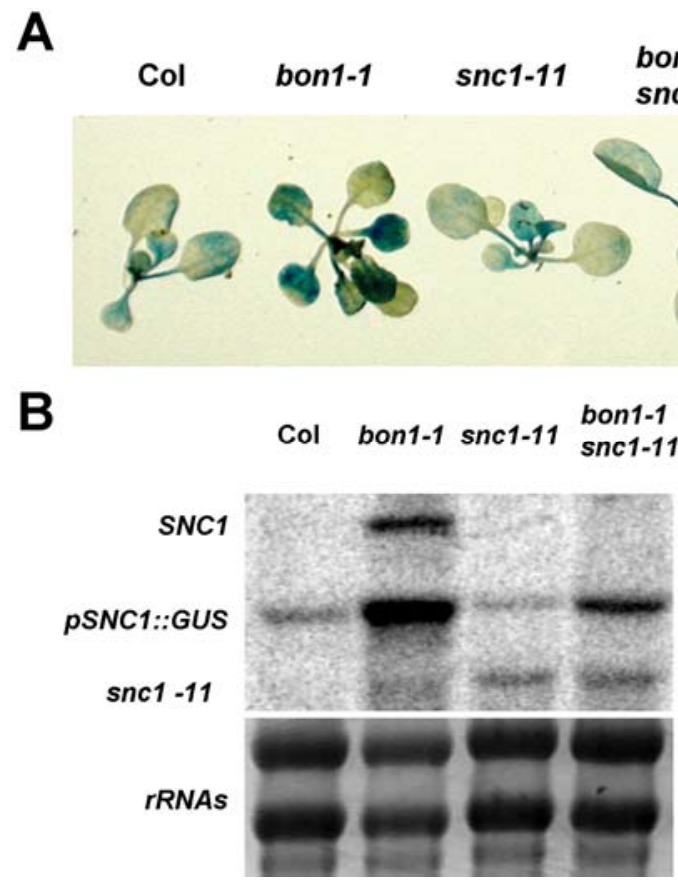

Fig. 6. $S N C 1$ is transcriptionally regulated by $S N C 1$ and BON1. A, Expression of $p S N C 1::$ GUS in near isogenic lines (NILs) in Col, bon1-1, snc1-11, and bon1-1snc1-11 background for a representative line assayed by $\beta$-glucuronidase (GUS) staining. B, Expression of $p S N C 1:: G U S$ and the endogenous SNC1 in NILs in Col, bon1-1, snc1-11, and bon1-1snc1-11 backgrounds for a representative line assayed by RNA blot analysis. A $S N C l$-specific probe hybridizes to the endogenous gene $S N C l$, the transgene, and the smaller $S N C 1$ transcript in snc1-11. cprl-1 was shown to be a possible sncl epi-allele (Stokes and Richards 2002) with a high expression of SNC1 (Yang and Hua 2004). Because there is no nucleotide sequence alteration of SNCl in sncl-2 or cprl-1, we could not identify the bonl1snc1-2 or bon1-1cprl-1 double mutants via genotyping F2 plants from crosses between bonl-1 and sncl-2 or between bon 1-1 and cpr1-1. However, we observed plants smaller than the singles in both populations at $22^{\circ} \mathrm{C}$ at a frequency of approximately $1 / 16$ and dwarf plants also were observed in F2 plants grown at $28^{\circ} \mathrm{C}$ (data not shown). Thus, it is likely that both the bon1-1snc1-2 and the bon1-1cpr1-1 doubles have more severe phenotypes than their respective single mutants, and further experiments will confirm the identities of the potential double mutants.

\section{DISCUSSION}

Here, we report the investigation the regulation of a TIRNB-LRR type of $R$ gene, $S N C 1$, at the transcript level and the mode of regulation of $S N C 1$ by its negative regulator, $B O N 1$. $S N C 1$ is a haplotype-specific gene found in the Col-0 accession of $A$. thaliana. When it is integrated into an exogenous chromosomal location as a transgene, it induces a dwarf phenotype likely due to an upregulation of defense response, indicating an auto-activation of the $S N C 1$ gene. With the use of the GUS reporter gene, our study indicates three levels of transcriptional regulation on $S N C 1$ : a repression by the chromosomal structure, a feed-back amplification from $S N C 1$, and a repression by $B O N 1$.

$S N C 1$ is under a negative control at its endogenous location. This is demonstrated by the higher expression of $p S N C 1:: G U S$ compared with the endogenous $S N C 1$ in transgenic plants and
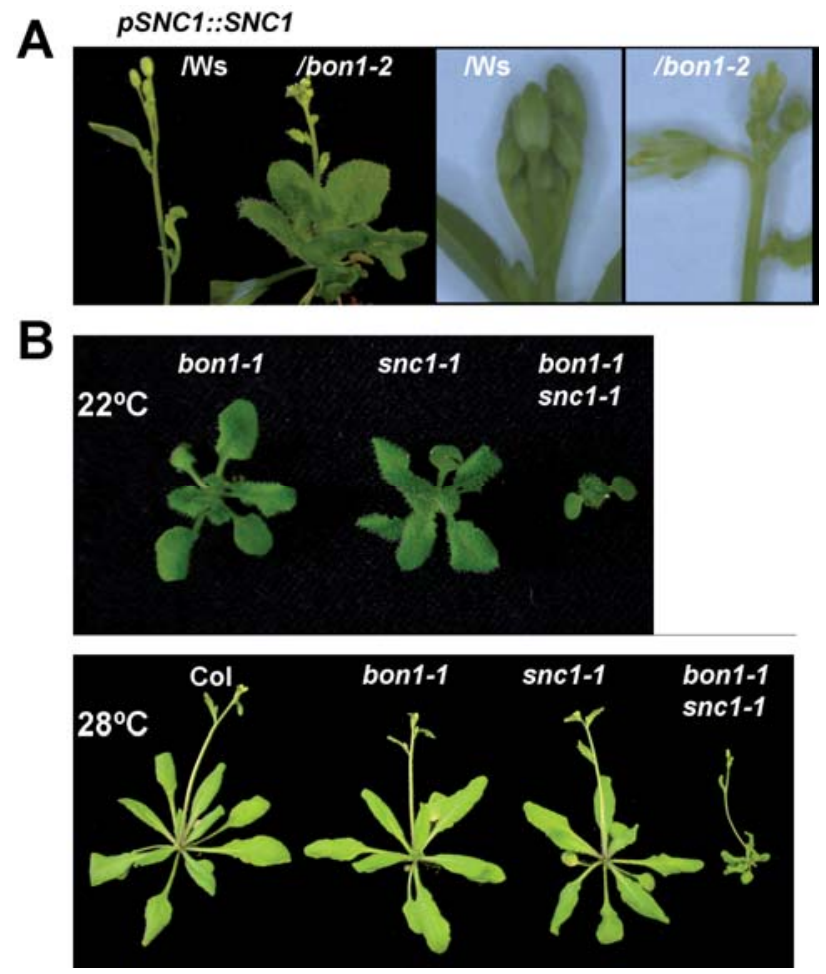

Fig. 7. Interaction among different regulations on $S N C 1$. A, Inflorescences of $p S N C 1: \because S N C 1 / \mathrm{Ws}$ and $p S N C 1: \because S N C 1 /$ bon $1-2$. Images in the black background (left) and those in the blue background (right) are of the same magnification. B, Interaction of sncl-1 and bon 1-1; bon 1-1 sncl-1 has a more severe phenotype than the single mutants at both 22 and $28^{\circ} \mathrm{C}$. Note that, at $28^{\circ} \mathrm{C}$, the single mutants are wild-type looking whereas the double mutants exhibit a dwarf phenotype. 
the wild type. Consistently, $p S N C 1:: S N C 1: G F P$ as a transgene has a higher expression than the endogenous $S N C 1$, even at $28^{\circ} \mathrm{C}$. We propose that the expression of $S N C 1$ is under a negative control at its endogenous location, possibly through chromatin structure or DNA methylation modification. The discovery of epi-alleles of $S N C 1$ supports the hypothesis that $S N C 1$ expression is repressed by its chromatin structure or a high methylation status (Stokes et al. 2002). SNC1 is located in a complex locus containing repeats of TIR-NB-LRR genes with homology to each other (Noel et al. 1999). In addition, two retrotransposon elements are embedded in this gene cluster. Both gene repeats and transposable elements could be the source of epigenetic control. The gene $b a l$, an epi-allele with high $S N C 1$ expression, was identified from a $d d m 1$ background, suggesting that demethylation may have induced an open chromatin structure for the transcription of SNC1. Two other potential SNCl epi-alleles are ssil and crpl, and their existence further supports the hypothesis that this gene is subject to epigenetic controls (Stokes and Richards 2002). It is very likely that when $S N C l$ is integrated into another chromosomal location, as in $p S N C 1:: S N C 1$ transgenic plants, repression normally imposed by its endogenous chromatin structure is lost and the exogenous location could assume an open chromatin structure for transcription.

SNC1 is under a feedback amplification through its promoter sequences. This was suggested in the bonl-1 study, where an upregulation of the SNCI transcript is dependent on the SAmediated defense responses (Yang and Hua 2004). This study shows that one or two copies of the SNCl transgene (even in the absence of the endogenous $S N C l$ gene, as in the Ws accession) can lead to upregulation of defense responses and a high $S N C 1$ expression. This is in contrast to previous studies, where several $R$ genes were shown to induce constitutive defense response. In those cases, $R$ genes either were expressed under an exogenous strong promoter such as the CaMV35S promoter, or required multiple copies of the transgene with its endogenous promoter (Oldroyd and Staskawicz 1998; Tang et al. 1999; Wulff et al. 2004; Xiao et al. 2003). Furthermore, $S N C 1$ expression in $p S N C 1:: S N C 1$ transgenic is higher under conditions inducing a dwarf phenotype than conditions conferring a wild-type phenotype, suggesting a feedback from the downstream defense. We generated transgenic lines of three other TIR-NB-LRR types of $R$ genes in the same RPP5 cluster under their native promoters and found that none of them induces dwarfism (unpublished data). The specific property of the SNC1 transgene supports an SNC1-specific autoactivation activity through its promoter. Alternatively, SNC1 may have a specific activity tied to growth control in addition to defense response control.

Using $p S N C 1:: G U S$ transgenics, we show that the feedback could be mediated by the $S N C 1$ promoter. $p S N C 1:: G U S$ has a higher expression in the wild-type $S N C 1$ background than in sncl-11, indicating that $S N C 1$ can positively regulate its own promoter. The overexpression of SNC1 in $p S N C 1:: S N C 1$ transgenics likely is due to a feedback amplification through the promoter sequences. This feedback amplification appears to be essential for the autoactivation of SNCl because overexpression by the CaMV $35 \mathrm{~S}$ promoter is less potent than the $S N C 1$ promoter in inducing autoactivation. A smaller proportion of p35S::SNC1 transgenic lines segregated dwarf phenotypes at a lower frequency when compared with $p S N C 1:: S N C 1$ transgenics (unpublished results). This suggests that it requires many copies of the transgene to drive extremely high expression of $S N C 1$ to induce activation in $p 35 S:: S N C 1$ transgenics. Similar feedback amplification of $R$ genes has been reported for SSI4 and $R P W 8$, and they are essential to generate the hypersensitive cell death phenotype induced by the $R P W 8$ transgene
(Shirano et al. 2002; Xiao et al. 2003). Autoactivation of SNC1 may involve a similar mechanism. An increase in expression of the $p S N C 1:: S N C 1$ transgene could slightly upregulate defense response which further enhances the expression of SNC1 through the $S N C 1$ promoter.

$S N C 1$ appears to be repressed at the transcriptional level by the BON1 gene. pSNC1::GUS expression is higher in bon 1-1 than in wild-type BON1. Using sncl-11 to block the feedback regulation, $p S N C 1:: G U S$ still has a higher expression in bon11 snc1-11 than in snc1-11. Thus, it appears that BON1 can negatively regulate $S N C 1$ at the transcriptional level. This regulation is likely to be indirect because BON1 is mostly localized to the plasma membrane and its associated protein BAP1 is mostly membrane bound. Transcriptional control by BON1 could be relayed by molecules generated with the perturbation of homeostasis with the loss of BON1 function. However, caution has to be taken to interpret these data because pSNC1:: GUS may not mimic endogenous $S N C l$ at the transcriptional control due to the release of local repression of the transgene. It also is yet to be determined whether this transcriptional regulation is the primary and sole mode of BON1 regulation of SNC1.

This study further suggests that different transcriptional controls might work in parallel. Although it has yet to be confirmed by comparing NILs, $p S N C 1:: S N C 1$ in general has a much more severe defect in bon 1-2 than in the wild-type Ws, especially in flower development. Derepression of $p S N C 1:: S N C 1$ from its endogenous chromosomal region in Ws likely is further enhanced by an upregulation of the SNC1 activity from the bonl2 mutation, indicating that these two regulations are distinct or at least not totally overlapping. Gene bon $1-1$ has a synergistic effect with snc1-1, suggesting that the mode of BON1 repression of $S N C 1$ is different from the protein activation or derepression by the snc1-1 missense mutation. Also, bon 1-1 appears to have a synergistic effect on sncl-2/bal, suggesting that $B O N 1$ regulation and epigenetic control of $S N C 1$ are likely different.

In sum, the regulation of $S N C 1$ possibly exemplifies a universal complex control of $R$ genes. In addition to the recent discoveries of $R$ activity regulation at the protein level, such as posttranslational modification, subcellular localization, and protein-protein interactions, $R$ activity also can be regulated at the RNA transcript level, as is found for $S N C 1$. These sophisticated regulations likely ensure a prevention of fitness cost by $R$ activation under nonstress conditions and a robust activation of defense response once the $R$ gene is induced.

\section{MATERIALS AND METHODS}

\section{Plant growth conditions.}

A. thaliana plants were grown at 22 or $28^{\circ} \mathrm{C}$ under constant light or a photoperiod of $12 \mathrm{~h}$ of light at $100 \mu \mathrm{mol} \mathrm{m} \mathrm{m}^{-2} \mathrm{~s}^{-1}$, with 50 to $70 \%$ relative humidity. Seed either were grown on petri dishes containing half-strength Murashige-Skoog medium (Sigma, St. Louis) supplemented with $2 \%$ sucrose and $0.7 \%$ agar or potted in soil (Metro-Mix 200; Sungro).

\section{RNA and DNA blot analyses.}

Total RNA was extracted from 3-week-old plants with the TRI Reagents (Molecular Research Inc., Ohio, U.S.A.) according to the manufacturer's protocol. RNA $(20 \mu \mathrm{g})$ was resolved on a $1 \%$ agarose gel containing $1.8 \%$ formaldehyde (Sambrook et al. 1989). Ethidium bromide was used to visualize the rRNA bands. For Southern blot analysis, genomic DNA was digested with $P v u I I$ and $B a m \mathrm{HI}$, electrophoresed through a $1 \%$ agarose gel, and blotted onto nylon membranes ((Hybond Hybond-N; Amersham Biosciences, Buckinghamshire, U.K.). RNA and 
DNA gel blots were hybridized with gene-specific, ${ }^{32} \mathrm{P}$-labeled, single-stranded DNA probes amplified by PCR except that only one primer was used to synthesize the antisense strand. Primers used for amplification of the probe template were as follows: SNC1-1 (5'ATATGGAGATAGCTTCTTCTTCTG) and SNC1-4 (5'AAGATCATATCCGGCCATAACTG) for SNC1 and PR1-1 (5'CTTTGTAGCTCTTGTAGGTGCTCT) and PR1-2 (5'TCCTGCATA-TGATGCTCCTTATTGA) for PRl.

\section{Plasmid construction and plant transformation.}

A 7.8-kb PstI genomic fragment containing the $S N C 1$ promoter and the coding region was cloned from BAC clone F5D3 (Arabidopsis Biological Resource Center, Columbus, OH, U.S.A.) into the binary vector pCAMBIA1300 (CAMBIA, Canberra, Australia) to generate the $\mathrm{p} S N C 1:: S N C 1$ construct.

A 6-kb genomic fragment containing the $S N C 1$ coding region and $3^{\prime}$ untranslated region was cloned from the BAC clone F5D3 into the binary vector pGreen-0229 (Hellens et al. 2000) to generate the $p 35 S:: S N C 1$ construct.

The pSNC1::GUS construct was generated by cloning a SNC1 genomic fragment with $1,904 \mathrm{bp} 5^{\prime}$ of the translation start codon ATG to 427 bp $3^{\prime}$ of ATG into the polylinker region of the binary vector PZP212 (Diener et al. 2000) in frame with the GUS gene cassette.

To construct the pSNC1::SNC1:GFP chimeric gene, a StuI site was added to the genomic fragment of $S N C 1$ before the stop codon of SNC1 via PCR. An EcoRV-StuI fragment of this product was ligated in frame with GFP flanked by $S t u$ I and PstI sites to create a $3^{\prime} S N C 1: G F P$ construct. The PstI-EcoRV fragment of the 5' $S N C 1$ from the $p S N C 1:: S N C 1$ construct and the EcoRV-PstI fragment of $3^{\prime}$ SNCl:GFP from the $3^{\prime}$ SNC1:GFP construct were ligated to the PstI site of pCAMBIA1300 to generate the pSNC1::SNC1:GFP construct.

Agrobacterium tumefaciens strain GV3101 (Koncz and Schell 1986) carrying different constructs was used to transform Arabidopsis plants via floral dip transformation (Clough and Bent 1998).

\section{Pathogen resistance assay.}

Bacterial growth in Arabidopsis was monitored as described (Yang et al. 2006). P. syringae pv. tomato DC3000 was grown overnight on the King's B medium and resuspended at $10^{8}$ $\mathrm{CFU} \mathrm{ml} \mathrm{m}^{-1}$ in a solution of $10 \mathrm{mM} \mathrm{MgCl}_{2}$ and $0.02 \%$ Silwet L77. Two-week-old seedlings were dip inoculated with bacteria and kept covered for $1 \mathrm{~h}$. The amount of bacteria in plants was analyzed at $1 \mathrm{~h}$ after dipping (day 0 ) and 3 days after dipping (day 3). The aerial parts of three inoculated seedlings were pooled for each sample and three samples were collected for each genotype at one time point. Seedlings were ground in 1 $\mathrm{ml}$ of $10 \mathrm{mM} \mathrm{MgCl}$ and serial dilutions of the ground tissue were used to determine the number of CFU per gram of leaf tissues.

\section{Analysis of GUS activity.}

Histochemical detection of GUS activity was performed as described (Hua et al. 2001). Tissues for GUS staining were incubated in staining solution $(50 \mathrm{mM}$ sodium phosphate, $\mathrm{pH}$ 7.0, $10 \mathrm{mM}$ EDTA, 2 mM 5-bromo-4-chloro-3-indoyl glucuronide, $1 \mathrm{mM}$ potassium ferricyanide, and $1 \mathrm{mM}$ potassium ferrocyanide) at $37^{\circ} \mathrm{C}$ overnight. After incubation, stained tissues were cleared of chlorophyll in an ethanol series.

\section{Generation of NILs.}

NILs of $p S N C 1:: G U S$ were isolated from $\mathrm{F} 2$ or $\mathrm{F} 3$ progenies of crosses between pSNC1::GUS/Col lines and bon1-1snc1-11 by PCR-based genotyping. Amplification by a T-DNA primer with a gene-specific primer indicates the presence of a mutant
T-DNA allele, whereas amplification by two gene-specific primers indicates the presence of a wild-type allele. Primers used for snc1-11 were wild-type allele: SNC1-1 and SNC1-4, and T-DNA mutant allele: SNC1-1 and JMLB1 (5'GGCAAT CAGCTGTTGCCCGTCTCACTGGTG). Primers for bon 1-1 were wild-type allele: MIN5 (5'ACGTTGAAAAGGGCACTG GTGTATG) and MINRIV319 (5'ATCGAATTCGTGGACAA GTTTACCGAGAC) and T-DNA mutant allele: MIN5 and LB5 (5'TGCTGATCCATGTAGATTTCC).

\section{ACKNOWLEDGMENTS}

Y. Li and S. Yang contributed equally to this work. This work is supported by the National Science Foundation under grant no. 0415597 to J. Hua. We thank ABRC for DNA clones and X. Dong and X. Li for mutant seed.

\section{LITERATURES CITED}

Belkhadir, Y., Subramaniam, R., and Dangl, J. L. 2004. Plant disease resistance protein signaling: NBS-LRR proteins and their partners. Curr. Opin. Plant Biol. 7:391-399.

Burch-Smith, T. M., Schiff, M., Caplan, J. L., Tsao, J., Czymmek, K., and Dinesh-Kumar, S. P. 2007. A novel role for the TIR domain in association with pathogen-derived elicitors. PLoS Biol. 5:e68.

Dangl, J. L., and Jones, J. D. 2001. Plant pathogens and integrated defence responses to infection. Nature 411:826-833.

Deslandes, L., Olivier, J., Peeters, N., Feng, D. X., Khounlotham, M., Boucher, C., Somssich, I., Genin, S., and Marco, Y. 2003. Physical interaction between RRS1-R, a protein conferring resistance to bacterial wilt, and PopP2, a type III effector targeted to the plant nucleus. Proc. Natl. Acad. Sci. U.S.A. 100:8024-8029.

Diener, A. C., Li, H., Zhou, W., Whoriskey, W. J., Nes, W. D., and Fink, G. R. 2000. Sterol methyltransferase 1 controls the level of cholesterol in plants. Plant Cell 12:853-870.

Dodds, P. N., Lawrence, G. J., Catanzariti, A. M., Teh, T., Wang, C. I., Ayliffe, M. A., Kobe, B., and Ellis, J. G. 2006. Direct protein interaction underlies gene-for-gene specificity and coevolution of the flax resistance genes and flax rust avirulence genes. Proc. Natl. Acad. Sci. U.S.A. 103:8888-8893.

Flor, H. H. 1971. Current status of the gene-for-gene concept. Annu. Rev. Phytopathol. 9:275-296.

Gu, K., Yang, B., Tian, D., Wu, L., Wang, D., Sreekala, C., Yang, F., Chu, Z., Wang, G. L., White, F. F., and Yin, Z. 2005. $R$ gene expression induced by a type-III effector triggers disease resistance in rice. Nature 435:1122-1125.

Hammond-Kosack, K. E., and Jones, J. D. 1996. Resistance gene-dependent plant defense responses. Plant Cell 8:1773-1791.

Heil, M., and Baldwin, I. T. 2002. Fitness costs of induced resistance: emerging experimental support for a slippery concept. Trends Plant Sci. 7:61-67.

Hellens, R. P., Edwards, E. A., Leyland, N. R., Bean, S., and Mullineaux, P. M. 2000. pGreen: a versatile and flexible binary Ti vector for Agrobacterium-mediated plant transformation. Plant Mol. Biol. 42:819-832.

Hua, J., Grisafi, P., Cheng, S. H., and Fink, G. R. 2001. Plant growth homeostasis is controlled by the Arabidopsis BON1 and BAP1 genes. Genes Dev. 15:2263-2272.

Jambunathan, N., Siani, J. M., and McNellis, T. W. 2001. A humidity-sensitive Arabidopsis copine mutant exhibits precocious cell death and increased disease resistance. Plant Cell 13:2225-2240.

Jia, Y., McAdams, S. A., Bryan, G. T., Hershey, H. P., and Valent, B. 2000 Direct interaction of resistance gene and avirulence gene products confers rice blast resistance. EMBO (Eur. Mol. Biol. Organ.) J. 19:40044014.

Lawton, K., Weymann, K., Friedrich, L., Vernooij, B., Uknes, S., and Ryals, J. 1995. Systemic acquired resistance in Arabidopsis requires salicylic acid but not ethylene. Mol. Plant-Microbe Interact. 8:863-870.

Li, X., Clarke, J. D., Zhang, Y., and Dong, X. 2001. Activation of an EDS1-mediated $R$-gene pathway in the snc 1 mutant leads to constitutive, NPR1-independent pathogen resistance. Mol. Plant-Microbe Interact. 14:1131-1139.

Martin, G., Bogdanove, A., and Sessa, G. 2003. Understanding the functions of plant disease resistance proteins. Annu. Rev. Plant Biol. 54:2361.

Meyers, B. C., Kozik, A., Griego, A., Kuang, H., and Michelmore, R. W. 2003. Genome-wide analysis of NBS-LRR-encoding genes in Arabidopsis. Plant Cell 15:809-834. 
Noel, L., Moores, T. L., van der Biezen, E. A., Parniske, M., Daniels, M. J., Parker, J. E., and Jones, J. D. 1999. Pronounced intraspecific haplotype divergence at the RPP5 complex disease resistance locus of Arabidopsis. Plant Cell 11:2099-2112.

Oldroyd, G. E. D., and Staskawicz, B. J. 1998. Genetically engineered broad-spectrum disease resistance in tomato. Proc. Natl. Acad. Sci. U.S.A 95:10300-10305.

Parker, J. E., Coleman, M. J., Szabo, V., Frost, L. N., Schmidt, R., van der Biezen, E. A., Moores, T., Dean, C., Daniels, M. J., and Jones, J. D. 1997. The Arabidopsis downy mildew resistance gene RPP5 shares similarity to the toll and interleukin-1 receptors with $\mathrm{N}$ and L6. Plant Cell 9:879-894.

Peart, J. R., Mestre, P., Lu, R., Malcuit, I., and Baulcombe, D. C. 2005. NRG1, a CC-NB-LRR protein, together with N, a TIR-NB-LRR protein, mediates resistance against Tobacco mosaic virus. Curr. Biol. 15:968973.

Sambrook, J., Fritsch, E. F., and Maniatis, T. A. 1989. Molecular Cloning: A Laboratory Manual, 2nd ed. Cold Spring Harbor Laboratory Press, Cold Spring Harbor, NY, U.S.A..

Schneider, D. S. 2002. Plant immunity and film noir: what gumshoe detectives can teach us about plant-pathogen interactions. Cell 109:537-540.

Shen, Q. H., Saijo, Y., Mauch, S., Biskup, C., Bieri, S., Keller, B., Seki, H., Ulker, B., Somssich, I. E., and Schulze-Lefert, P. 2007. Nuclear activity of MLA immune receptors links isolate-specific and basal disease-resistance responses. Science 315:1098-1103.

Shirano, Y., Kachroo, P., Shah, J., and Klessig, D. F. 2002. A gain-of-function mutation in an Arabidopsis toll interleukin1 receptor-nucleotide binding site-leucine-rich repeat type $R$ gene triggers defense responses and results in enhanced disease resistance. Plant Cell 14:3149-3162.

Stokes, T. L., and Richards, E. J. 2002. Induced instability of two Arabidopsis constitutive pathogen-response alleles. Proc. Natl. Acad. Sci. U.S.A. 99:7792-7796.

Stokes, T. L., Kunkel, B. N., and Richards, E. J. 2002. Epigenetic variation in Arabidopsis disease resistance. Genes Dev. 16:171-182.

Takken, F. L., Albrecht, M., and Tameling, W. I. 2006. Resistance proteins: molecular switches of plant defence. Curr. Opin. Plant Biol. 9:383-390.
Tang, X., Xie, M., Kim, Y. J., Zhou, J., Klessig, D. F., and Martin, G. B. 1999. Overexpression of Pto activates defense responses and confers broad resistance. Plant Cell 11:15-29.

Uknes, S., Mauch-Mani, B., Moyer, M., Potter, S., Williams, S., Dincher, S., Chandler, D., Slusarenko, A., Ward, E., and Ryals, J. 1992. Acquired resistance in Arabidopsis. Plant Cell 4:645-656.

van der Biezen, E. A., and Jones, J. D. 1998. Plant disease-resistance proteins and the gene-for-gene concept. Trends Biochem. Sci. 23:454-456.

van der Biezen, E. A., Freddie, C. T., Kahn, K., Parker, J. E., and Jones, J. D. 2002. Arabidopsis RPP4 is a member of the RPP5 multigene family of TIR-NB-LRR genes and confers downy mildew resistance through multiple signalling components. Plant J. 29:439-451.

Wulff, B. B., Kruijt, M., Collins, P. L., Thomas, C. M., Ludwig, A. A., De Wit, P. J., and Jones, J. D. 2004. Gene shuffling-generated and natural variants of the tomato resistance gene $C f-9$ exhibit different auto-necrosis-inducing activities in Nicotiana species. Plant J. 40:942-956.

Xiao, S., Brown, S., Patrick, E., Brearley, C., and Turner, J. G. 2003. Enhanced transcription of the Arabidopsis disease resistance genes $R P W 8.1$ and $R P W 8.2$ via a salicylic acid-dependent amplification circuit is required for hypersensitive cell death. Plant Cell 15:33-45.

Yang, H., Li, Y., and Hua, J. 2006. The C2 domain protein BAP1 negatively regulates defense responses in Arabidopsis. Plant J. 48:238248.

Yang, S., and Hua, J. 2004. A haplotype-specific Resistance gene regulated by BONZAI1 mediates temperature-dependent growth control in Arabidopsis. Plant Cell 16:1060-1071.

Zhang, Y., Goritschnig, S., Dong, X., and Li, X. 2003. A gain-of-function mutation in a plant disease resistance gene leads to constitutive activation of downstream signal transduction pathways in suppressor of npr 11, constitutive 1. Plant Cell 15:2636-2646.

\section{AUTHOR-RECOMMENDED INTERNET RESOURCE}

National Institute of Health ImageJ website: rsb.info.nih.gov/ij/ 